\title{
Cure and care for children and adolescents with acute myeloid leukemia in Middle and Eastern European countries: part II
}

\author{
Michael N. Dworzak
}

Received: 3 February 2014 / Accepted: 11 February 2014 / Published online: 4 March 2014 (C) Springer-Verlag Wien 2014

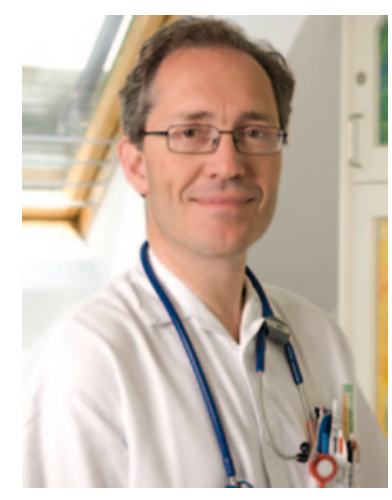

Michael N. Dworzak
Acute myeloid leukemia (AML), has always been among the most dreadful cancers of childhood and adolescence. Curing this type of leukemia still engages the most intense chemotherapy available in pediatrics, which also contains stem cell transplantation (SCT) in a considerable proportion of cases.

Recently, a valuable overview over the past and current standards, treatment results as well as challenges in the management of pediatric AML throughout several European countries including Czech Republic, Hungary, Serbia, Slovak Republic, and Poland was provided in Memo [1-6]. In this issue, further three European national pediatric hemato-oncology study groups (from Austria, Greece, and Slovenia) report their data-which were collected over a long observation period of 20 years-in a similar way [7-9]. All these, mostly population-based, results were generated using the Berlin-FrankfurtMünster (BFM) AML treatment backbone. Notably, the AML-BFM strategy evolved since 1978 by launching new treatment trials every 5-6 years [10]. For each new trial, the treatment backbone was adapted based on the experience gained from previous own studies as well as from those of other study groups. Along with continuously improving diagnostic procedures, application of elaborate supportive care, as well as case management only in dedicated, highly experienced centers, this strategy led to significantly increased cure rates in an international context.

Hence-as can be deduced from the data published in Memo-current proportional benchmarks for the cure

Assoc. Prof. M. N. Dworzak, MD, PhD ( $\triangle)$

AML-BFM Study-Chair Austria, St. Anna Children's Hospital and Children's Cancer Research Institute, Department of Pediatrics,

Medical University of Vienna,

Kinderspitalgasse 6, 1090 Vienna, Austria

e-mail:dworzak@stanna.at and care of children and adolescents with AML in Europe can be formulated: (i) the cumulative nonleukemic death rate (including early deaths due to hemorrhage or acute respiratory distress syndrome, deaths in complete remission, e.g., due to infections, and treatment-related mortality of SCT) should be less than $10 \%$ of all patients; (ii) there should be less than $35 \%$ of patients suffering from a relapse and more than $65 \%$ overall should be cured (the latter may include about $10 \%$ of all patients who can be cured after a first relapse); (iii) about $90 \%$ of patients with lower-risk genetics (i.e., up to $35 \%$ of all pediatric AMLs as characterized by translocations PML-RARA, RUNX1-RUNX1T1 (AML1-ETO), CBFB-MYH11, or by GATA-1 mutation) should be long-term surviving; (iv) at least one SCT is needed in another group of about $35 \%$ of all patients-many but not all of whom can nowadays be predefined based on diagnostic algorithms-to safeguard a chance of cure, but this unfortunately remains without a guarantee of long-term success.

Academic treatment trials and the dedicated input of involved stakeholders have led to these respectable improvements in the outcome of a disease, which was considered mostly incurable just 40 years ago. But driving cure rates further up until most-if not all-children with AML are to be saved will need extraordinary input not only of academia and clinical teams, but also of society, regulators, and pharmaceutical industry. Undoubtedly, there are important societal implications when it comes to financing both, standard care with appropriate diagnostics and the validated treatment backbone (which is available as outlined by the plenitude of data now published also herein), as well as research including data registration, observation, and analysis for the benefit of patients [11]. Some obstacles to this end need to be turned down by the regulators themselves because they are just created by current regulations or their interpretation. For example, it will be most important for the understanding of the quality of care to reg- 
ister all those patients who present first as emergency case or even die very early irrespectively of whether or not they enter a clinical trial. Clearly, this is problematic with respect to the need of obtaining informed consent for trial participation in an emergency situation. Hence, not being able to obtain written consent before event occurrence may lead to important selection bias. Hence, trial policies permitting waivers of prior informed consent for minimal-risk observational research are urgently needed as are policies that allow a better linkage of registries with randomized treatment trials on top [12]. This may allow observing all diseased patients and their treatment course based on a standard backbone therapy upon inclusion into low-cost observational registries toward composite end-points. In addition, this may allow us to concomitantly conduct randomized assessments of specific questions (with a higher degree of risk) among subcohorts of patients of the registry for a limited period of observation [13]. A better understanding of the issues at the regulator level may lead to more appropriate design and interpretation of regulations for academia-driven clinical trials. Eventually, this should lead to the expectation of the society that participation in clinical registries complemented with randomized treatment optimization trials is the norm rather than the exception when it comes to care for children and adolescents with cancer [14].

This latter aspect is particularly momentous when realizing that four out of the six most important chemotherapeutic drugs used in the AML-BFM backbone treatment (which allow for the respectable cure rates of children with AML delineated herein) are still not licensed for the treatment of children and adolescents in Europe (i.e., mitoxantrone, idarubicin, liposomal daunorubicin, and 2-CdA). Over those 35 years of clinical trials, however, dedicated work of academia has elaborated a surplus of information on outcome end-points and cumulative toxicities when using these drugs in combinations throughout all age groups of childhood, with no chance to generate an impact on market authorization and labeling. It was clearly shown that those off-label drugs save more lives than could be saved by using just drugs with market authorization [1519]. Hence, the question of label should be separated from market authorization and economic interests by creating an evidence-based clinical application label. One can definitely accept that the current AML-BFM backbone has produced sufficient evidence to consider those six most important drugs included as "labeled" for curing children and adolescents with AML. And in that context, despite being off-label with respect to marketing, these established chemotherapeutic drugs and their combinations in the AML-BFM backbone do not need to be considered investigational medicinal products sensu strictu when new trials for treatment optimization are to be submitted to dedicated authorities and ethics boards.

In conclusion, we have come far in caring for and curing children and adolescents with AML_-but we need significant joint efforts of all stakeholders including also society, regulators, and even the pharmaceutical industry and the willingness for a change-to come even farer toward our goal which is curing all children and adolescents with AML.

\section{Conflict of interest}

The author declares that there is no conflict of interest.

\section{References}

1. Creutzig U, Tallen G. Treatment results for children and adolescents with acute myeloid leukemia in middle and eastern European countries. Memo. 2013;6:5-8.

2. Sramkova L, Sterba J, Hrstkova H, et al. Development of treatment and clinical results in childhood acute myeloid leukemia in the Czech Republic. Memo. 2013;6:41-5.

3. Kolenova A, Bubanska E, Oravkinova I, et al. Development of treatment and clinical results in childhood acute myeloid leukemia in the Slovak Republic. Memo. 2013;6:46-53.

4. Balwierz W, Pawinska-Wasikowska K, Klekawka T, et al. Development of treatment and clinical results in childhood acute myeloid leukemia in Poland. Memo. 2013;6:54-62.

5. Krstovski N, Kuzmanovic M, Vujic D, et al. Treatment results of childhood acute myeloid leukemia in Serbia. Memo. 2013;6:63-8.

6. Szegedi I, Jakab Z, Masath P, et al. Development of treatment and clinical results in childhood acute myeloid leukemia in Hungary. Memo. 2013;6:69-72.

7. Boztug H, Mühlegger N, Glogova E, et al. Development of treatment and clinical results in childhood AML in Austria (1993-2013). Memo. 2014;7(1). doi:10.1007/ s12254-014-0135-y.

8. Polychronopoulou S, Baka M, Servitzoglou M, et al. Treatment and clinical results in childhood AML in Greece. Memo. 2014;7(1). doi:10.1007/s12254-013-0112-x.

9. Velikonja O, Stefanović M, Kitanovski L, et al. Development of treatment and clinical results in childhood acute myeloid leukemia in Slovenia in the period from 1991 to 2010. Memo. 2014;7(1). doi:10.1007/s12254-013-0090-z.

10. Creutzig U, Zimmermann M, Dworzak MN, et al. Development of a curative treatment within the AML-BFM studies. Klin Padiatr 2013;225 (Suppl. 1):79-86.

11. Tallen G, Dworzak M, Gadner H, et al. Imperative of continual support by the European community for future advances in paediatric oncology in Europe: meeting report of the EC-funded sciencecommunication project DIRECT “Overcoming Cancer with Research". Memo. 2009;2:234-45.

12. Tu JV, Willison DJ, Silver FL, et al. Impracticability of informed consent in the registry of the Canadian stroke network. N Engl J Med. 2004;350:1414-21.

13. Lauer MS, D'Agostino RB. The Randomized registry trialthe next disruptive technology in clinical research? N Engl J Med. 2013;369:1979-81.

14. Reith C, Landray M, Devereaux PJ, et al. Randomized clinical trials-removing unnecessary obstacles. N Engl J Med. 2013;369:1061-65.

15. Creutzig U, Ritter J, Zimmermann M, et al. Improved treatment results in high-risk pediatric acute myeloid leukemia patients after intensification with high-dose cytarabine and mitoxantrone: results of study acute myeloid leukemia-Berlin-Frankfurt-Munster 93. J Clin Oncol. 2001;19(10):2705-13. 
16. Creutzig U, Ritter J, Zimmermann M, et al. Idarubicin improves blast cell clearance during induction therapy in children with AML: results of study AML-BFM 93. AMLBFM Study Group. Leukemia. 2001;15(3):348-54.

17. Creutzig U, Zimmermann M, Bourquin JP, et al. Second induction with high-dose cytarabine and mitoxantrone: different impact on pediatric AML patients with $\mathrm{t}(8 ; 21)$ and with inv(16). Blood. 2011;118(20):5409-15.

18. Creutzig U, Zimmermann M, Bourquin JP, et al. Randomized trial comparing liposomal daunorubicin with idarubicin as induction for pediatric acute myeloid leukemia: results from study AML-BFM 2004. Blood. 2013;122(1):37-43.
19. Kaspers GJ, Zimmermann M, Reinhardt D, et al. Improved outcome in pediatric relapsed acute myeloid leukemia: results of a randomized trial on liposomal daunorubicin by the International BFM Study Group. J Clin Oncol. 2013;31(5):599-607. 